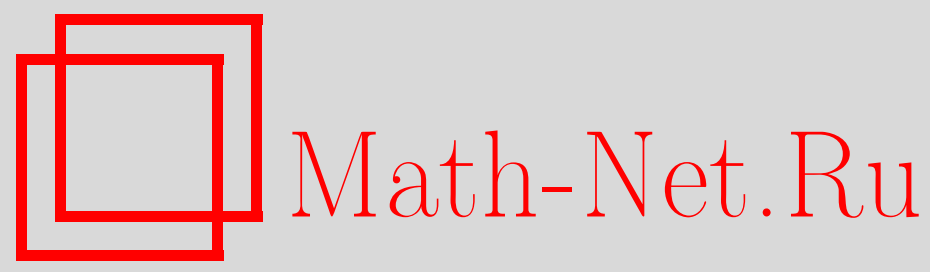

А. Е. Мамонтов, Оценки глобальной регулярности для многомерных уравнений сжимаемой неньютоновской жидкости, Матем. заметки, 2000, том 68, выпуск 3, 360-376

DOI: https://doi.org/10.4213/mzm953

Использование Общероссийского математического портала Math-Net.Ru подразумевает, что вы прочитали и согласны с пользовательским соглашением http://www.mathnet.ru/rus/agreement

Параметры загрузки:

IP : 52.6 .47 .48

26 апреля 2023 г., 15:08:39

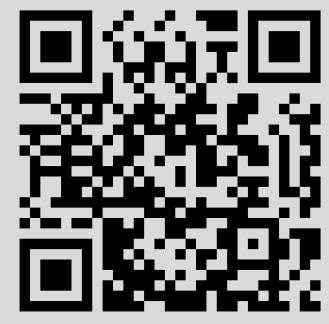




\section{ОЦЕНКИ ГЛОБАЛЬНОЙ РЕГУЛЯРНОСТИ ДЛЯ МНОГОМЕРНЫХ УРАВНЕНИЙ СЖИМАЕМОЙ НЕНЬЮТОНОВСКОЙ ЖИДКОСТИ}

\section{А. Е. Мамонтов}

Работа посвящена вопросу существования "в целом" достаточно регулярных решений двух- и трехмерных уравнений сжимаемой нелинейно-вязкой жидкости. Для случая потенциального тензора напряжений разработана техника вывода энергетических тождеств, не содержаших производных от плотности. На основе этих тождеств для достаточно быстро растущих потенциалов получена развернутая система априорных оценок для указанных уравнений. Также рассматривается смежный вопрос об оценке решений нелинейной эллиптической системы, связанной с тензором напряжений.

Библиограбфия: 11 названий.

В данной статье изучается система уравнений, описьвающая движение вязких сжимаемых жидкостей (без учета тепловых явлений), которую мы запишем в следующем виде:

$$
\begin{aligned}
& \frac{d \rho}{d t}+\rho \operatorname{div} \boldsymbol{u}=0, \\
& \frac{d \boldsymbol{u}}{d t}=\boldsymbol{w}+\boldsymbol{f}, \\
& \operatorname{div} \mathbb{P}=\rho \boldsymbol{w} .
\end{aligned}
$$

Здесь $\rho$ - плотность жидкости, $\boldsymbol{u}$ - вектор скорости, $\mathbb{P}$ - тензор напряжений, $\boldsymbol{w}$ - вспомогательный вектор (играющий в дальнейшем важную роль), $\boldsymbol{f}$ - вектор внешних массовых сил; все указанные величины являются функциями от времени $t$ и пространственных переменных $\boldsymbol{x} \in \mathbb{R}^{n}, n \geqslant 2$. При этом операторы $\operatorname{div}$ и $\nabla$ действуют по $\boldsymbol{x}, \mathrm{a}$

$$
\frac{d}{d t} \equiv \frac{\partial}{\partial t}+u \cdot \nabla
$$

(материальная производная). Величины $\rho$ и $\boldsymbol{u}$ искомые, $\boldsymbol{f}$ задано, а $\mathbb{P}$ задается заранее как некоторая функция от $\rho$ и $\boldsymbol{u}$; вид этой функции (назьваемой определяюшим уравнением для напряжений) зависит от конкретной физической модели. При этом требование инвариантности модели относительно системы координат приводит к ограничению класса определяющих уравнений изотропными связями, представимыми в виде [1]

$$
\mathbb{P}=\sum_{s \geqslant 0} \beta_{s}\left(\rho, J_{1}(\mathbb{D}), \ldots, J_{n}(\mathbb{D})\right) \mathbb{D}^{s},
$$


где $J_{i}(\mathbb{D})$ суть основные инварианты тензора скоростей деформаций $\mathbb{D}$ (определен ниже), а $\beta_{s}$ - некоторые функции. Обычно в (0.5) пишут сумму по $s \leqslant n-1$ (это не меняет класса в силу теоремы Гамильтона-Кэли), однако нам удобнее оставить высшие степени для более прозрачной формулировки свойств коэффициентов $\beta_{s}$, которую мы сделаем позднее.

Если связь (0.5) линейна по отношению к $\mathbb{D}$, т.е. имеет вид

$$
\mathbb{P}=(-p(\rho)+\beta(\rho) \operatorname{tr} \mathbb{D}) \mathbb{I}+\gamma(\rho) \mathbb{D}
$$

(I - единичный тензор, $p$ - давление, при этом, как правило, так назьваемые коэффициенты вязкости $\beta$ и $\gamma$ полагают постоянными), то говорят, что (0.1)-(0.5) представляет собой модель ньютоновской жидкости, иначе - неньютоновской. Математическая теория как тех, так и других содержит множество нерешенных вопросов, начиная от проблемы корректности в целом по времени и входным данньм, для которой (в случае $n \geqslant 2$ ) либо нет удовлетворительного решения, либо делаются первые шаги в этом направлении. Для ньютоновской модели упомянем работу [2], где доказана глобальная корректность при $n=2$, и монографию [3], в которой изучаются глобальные (но “слабые” ) решения при произвольном $n$. Изучениененьютоновских моделей стимулируется, таким образом, помимо собственного интереса еще и поиском путей к построению глобальной теории для системы (0.1)-(0.4) вообще. Современное состояние математической теории неньютоновских жидкостей отражено в монографии [4], где изучаются достаточно подробно только однородные несжимаемые жидкости (т.е. модели с $\rho \equiv$ const), сжимаемые же почти не рассматриваются (получены только весьма слабые мерозначные решения). В более сильных классах разрешимость уравнений (0.1)-(0.4) доказана (при произвольном $n$ ) в работах [5]-[7], однако вопрос о повьшении гладкости построенных решений по-прежнему открыт. Этому вопросу и посвящена данная работа. Здесь мы существенно опираемся на технику оценок для уравнений Навье-Стокса сжимаемой жидкости, развитую в [2] для двумерных ньютоновских уравнений.

Уточним некоторые обозначения и соотношения. Символом $\mathbb{D}(\boldsymbol{a})$ будем обозначать тензор симметрической части производных векторного поля $\boldsymbol{a}$ :

$$
\mathbb{D}(\boldsymbol{a})=\operatorname{Sym}(\nabla \otimes \boldsymbol{a})=\frac{(\nabla \otimes \boldsymbol{a})+(\nabla \otimes \boldsymbol{a})^{*}}{2},
$$

т.е.

$$
D_{i j}(\boldsymbol{a})=\frac{1}{2}\left(\frac{\partial a_{i}}{\partial x_{j}}+\frac{\partial a_{j}}{\partial x_{i}}\right)
$$

причем для $\boldsymbol{a}=\boldsymbol{u}$ будем писать просто $\mathbb{D}$ (тензор скоростей деформаций). Символ “ $\otimes$ ” означает тензорное произведение: $(\boldsymbol{a} \otimes \boldsymbol{b})_{i j}=a_{i} b_{j}$.

В дальнейшем мы ограничимся определяющими уравнениями (для напряжений) вида

$$
\mathbb{P}=-p(\rho) \mathbb{I}+\frac{\partial V(\mathbb{D})}{\partial \mathbb{D}},
$$

где $V$ - так назьваемый потенциал, а $\partial V / \partial \mathbb{D}$ есть тензор с компонентами $\partial V(\mathbb{D}) / \partial D_{i j}$. Если $V-$ (скалярная) функция $\mathbb{D}$ как тензора (т.е. изотропная функция), то $\partial V / \partial \mathbb{D}$ действительно является тензором, т.е. изотропной функцией, и тогда (0.6) есть частньй случай (0.5) (однако в п. 1 и в начале п. 2 изотропность $V$ не требуется, достаточно лишь 
симметричности $\partial V / \partial \mathbb{D})$. Будем рассматривать также высшие производные $\partial^{2} V / \partial \mathbb{D}^{2}$ и $\partial^{3} V / \partial \mathbb{D}^{3}$ (представляющие собой тензоры четвертого и шестого ранга соответственно), трактуемые как билинейная и трилинейная формы над тензорами второго ранга:

$$
\begin{aligned}
\frac{\partial^{2} V}{\partial \mathbb{D}^{2}}\langle\mathbb{A}, \mathbb{B}\rangle & =\sum_{i, j, k, l=1}^{n} \frac{\partial^{2} V}{\partial D_{i j} \partial D_{k l}} A_{i j} B_{k l}, \\
\frac{\partial^{3} V}{\partial \mathbb{D}^{3}}\langle\mathbb{A}, \mathbb{B}, \mathbb{C}\rangle & =\sum_{i, j, k, l, \sigma, \tau=1}^{n} \frac{\partial^{3} V}{\partial D_{i j} \partial D_{k l} \partial D_{\sigma \tau}} A_{i j} B_{k l} C_{\sigma \tau} .
\end{aligned}
$$

Напомним стандартные обозначения для тензоров второго ранга:

$$
\mathbb{A}: \mathbb{B}=\sum_{i, j=1}^{n} A_{i j} B_{i j}, \quad|\mathbb{A}|=(\mathbb{A}: \mathbb{A})^{1 / 2}, \quad \operatorname{tr} \mathbb{A}=\mathbb{A}: \mathbb{I}
$$

(при этом отметим, что $|\cdot|$ является нормой в $\mathscr{L}\left(\mathbb{R}^{n}\right)$ и потому обладает всеми свойствами операторной нормы; в частности, $|\mathbb{A B}| \leqslant|\mathbb{A}| \cdot|\mathbb{B}|)$, и аналогично $|\cdot|$ для тензоров высокого ранга. В дальнейшем под словом “тензор” будем, если не оговорено противное, подразумевать тензор второго ранга.

1. Вывод энергетических тождеств: абстрактная схема. При выводе априорных оценок системы (0.1)-(0.4) фундаментальную роль играют так назьваемые энергетические тождества - дифференциальные уравнения (по $t$ ) для специальных функционалов от решения. Мы выводим их (в этом пункте) достаточно стандартным способом из системы (0.1)-(0.4), рассматриваемой как уравнения для величин $(\rho, \boldsymbol{u})$, и подходящим образом (а именно так, чтобы не возникали новые производные от плотности) построенных ее дифференциальных продолжений, рассматриваемых как уравнения для величин $(\rho, V),(\rho, \boldsymbol{w}),(\rho, \nabla \otimes \boldsymbol{w})$ и т.д. При этом основные операции (в том числе построение дифференциальных продолжений) производятся над уравнением импульса (0.2), а уравнение неразрьвности (0.1) и вспомогательное уравнение $(0.3)$ играют роль своеобразных связей, позволяющих исключать возникающие производные от $\rho$.

Прежде всего введем в рассмотрение скобки (для цельх $k \geqslant 0$ )

$$
[\mathbb{A}, \boldsymbol{a}]^{(k)}=\sum_{|\alpha|=k}\left(D_{\boldsymbol{x}}^{\alpha} \mathbb{A}\right)\left(D_{\boldsymbol{x}}^{\alpha} \boldsymbol{a}\right), \quad[\mathbb{A}, \boldsymbol{a}]_{(1)}=\sum_{i, j, k=1}^{n} \frac{\partial A_{i j}}{\partial x_{k}} \cdot \frac{\partial a_{k}}{\partial x_{j}} \boldsymbol{e}_{i}
$$

( $\boldsymbol{e}_{i}$ - базисные векторы, $\mathbb{A}$ - тензор, $\boldsymbol{a}$ - вектор, значения скобок - векторы), а также отметим правило коммутации операторов $\nabla$ и $d / d t$ :

$$
\nabla \frac{d H}{d t}=\frac{d}{d t} \nabla H+(\nabla \otimes \boldsymbol{u}) \nabla H
$$

( $H$-произвольная скалярная функция), из которого, в частности, следуют соотношения

$$
\begin{gathered}
\mathbb{D}\left(\frac{d \boldsymbol{u}}{d t}\right)=\frac{d \mathbb{D}}{d t}+\mathbb{C}, \quad \text { где } \mathbb{C}=\operatorname{Sym}\left((\nabla \otimes \boldsymbol{u})^{2}\right), \\
\operatorname{div} \frac{d \mathbb{A}}{d t}=\frac{d}{d t} \operatorname{div} \mathbb{A}+[\mathbb{A}, \boldsymbol{u}]_{(1)} .
\end{gathered}
$$


Предположим, что имеется достаточно гладкое решение системы $(0.1)-(0.4),(0.6)$ в цилиндре $(a, b) \times \Omega$ (где $\Omega$ - область в $\mathbb{R}^{n}$ с гладкой гранищей или параллелепипед), и выведем при $a<t<b$ энергетические тождества.

Умножив (0.1) на $|\boldsymbol{u}|^{2} / 2,(0.2)$ на $\rho \boldsymbol{u}$ и сложив, получим уравнение

$$
\frac{\partial}{\partial t}\left(\frac{\rho|\boldsymbol{u}|^{2}}{2}\right)+\operatorname{div}\left(\frac{\rho|\boldsymbol{u}|^{2}}{2} \boldsymbol{u}\right)=\operatorname{div}(\mathbb{P} \boldsymbol{u})-\frac{\partial V}{\partial \mathbb{D}}: \mathbb{D}+p \operatorname{div} \boldsymbol{u}+\rho \boldsymbol{u} \cdot \boldsymbol{f}
$$

Введя в рассмотрение функцию

$$
h(\xi)=\xi \int_{1}^{\xi} \eta^{-2} p(\eta) d \eta
$$

из (0.1) вьведем соотношение

$$
\frac{\partial h(\rho)}{\partial t}+\operatorname{div}(h(\rho) \boldsymbol{u})+p(\rho) \operatorname{div} \boldsymbol{u}=0
$$

сложив которое с (1.4) и проинтегрировав по $\Omega$, получим первое энергетическое тождество

$$
\begin{aligned}
& \frac{d}{d t} \int_{\Omega}\left(\frac{\rho|\boldsymbol{u}|^{2}}{2}+h(\rho)\right) d \boldsymbol{x}+\int_{\Omega} \frac{\partial V}{\partial \mathbb{D}}: \mathbb{D} d \boldsymbol{x} \\
& \quad=\int_{\Omega} \rho \boldsymbol{u} \cdot \boldsymbol{f} d \boldsymbol{x}+\int_{\partial \Omega}\left(\mathbb{P} \boldsymbol{u}-\left(\frac{\rho|\boldsymbol{u}|^{2}}{2}+h(\rho)\right) \boldsymbol{u}\right) \cdot \boldsymbol{n} d S
\end{aligned}
$$

Пользуясь $(1.2),(0.2)$ и (0.3), вьведем уравнение для $V$, преобразовав выражение

$$
\begin{aligned}
\operatorname{div}\left(\mathbb{P} \frac{d \boldsymbol{u}}{d t}\right) & =\mathbb{P}: \mathbb{D}\left(\frac{d \boldsymbol{u}}{d t}\right)+\operatorname{div} \mathbb{P} \cdot \frac{d \boldsymbol{u}}{d t}=\mathbb{P}: \frac{d \mathbb{D}}{d t}+\mathbb{P}: \mathbb{C}+\rho \boldsymbol{w} \cdot(\boldsymbol{w}+\boldsymbol{f}) \\
& =\frac{d V}{d t}-p \frac{d}{d t} \operatorname{div} \boldsymbol{u}+\mathbb{P}: \mathbb{C}+\rho|\boldsymbol{w}|^{2}+\rho \boldsymbol{w} \cdot \boldsymbol{f}
\end{aligned}
$$

Перебрасьвая оператор $d / d t$ c $\operatorname{div} \boldsymbol{u}$ на $p$ и учитьвая соотношение

$$
\frac{d p}{d t}=-\rho p^{\prime}(\rho) \operatorname{div} \boldsymbol{u}
$$

(следствие $(0.1))$, после интегрирования по $\Omega$ получим второе энергетическое тождество

$$
\begin{aligned}
& \frac{d}{d t} \int_{\Omega}(V-p \operatorname{div} \boldsymbol{u}) d \boldsymbol{x}+\int_{\Omega} \rho|\boldsymbol{w}|^{2} d \boldsymbol{x}=\int_{\Omega}(V-p \operatorname{div} \boldsymbol{u}) \operatorname{div} \boldsymbol{u} d \boldsymbol{x} \\
& \quad+\int_{\Omega}\left(\rho p^{\prime}(\rho)(\operatorname{div} \boldsymbol{u})^{2}-\rho \boldsymbol{w} \cdot \boldsymbol{f}-\mathbb{P}: \mathbb{C}\right) d \boldsymbol{x}+\int_{\partial \Omega}\left(\mathbb{P} \frac{d \boldsymbol{u}}{d t}-(V-p \operatorname{div} \boldsymbol{u}) \boldsymbol{u}\right) \cdot \boldsymbol{n} d S
\end{aligned}
$$

Если “исходить" от выражения

$$
\operatorname{div}\left(\mathbb{P} \frac{\partial u}{\partial t}\right)
$$


то нетрудно получить другой вариант этого тождества:

$\frac{d}{d t} \int_{\Omega} V d \boldsymbol{x}+\int_{\Omega} \rho|\boldsymbol{w}|^{2} d \boldsymbol{x}=\int_{\Omega} \rho \boldsymbol{w} \cdot((\boldsymbol{u} \cdot \nabla) \boldsymbol{u}-\boldsymbol{f}) d \boldsymbol{x}+\int_{\Omega} p \frac{\partial \operatorname{div} \boldsymbol{u}}{\partial t} d \boldsymbol{x}+\int_{\partial \Omega}\left(\mathbb{P} \frac{\partial \boldsymbol{u}}{\partial t}\right) \cdot \boldsymbol{n} d S$,

от которого, однако, можно ожидать пользы только в модели Бюргерса (т.е. при $p(\rho) \equiv 0)$ ввиду наличия в правой части "неустранимого" второго интеграла.

Выведем дифференциальное продолжение уравнения импульса (0.2), применив к нему операцию $\mathbb{D}(\cdot)$ с учетом $(1.2)$ :

$$
\frac{d \mathbb{D}}{d t}+\mathbb{C}=\mathbb{D}(\boldsymbol{w})+\mathbb{D}(\boldsymbol{f})
$$

и применим к (1.9) операцию $\frac{\partial^{2} V}{\partial \mathbb{D}^{2}}\langle\mathbb{D}(\boldsymbol{w}), \cdot\rangle$ :

$$
\frac{\partial^{2} V}{\partial \mathbb{D}^{2}}\langle\mathbb{D}(\boldsymbol{w}), \mathbb{D}(\boldsymbol{w})\rangle+\frac{\partial^{2} V}{\partial \mathbb{D}^{2}}\langle\mathbb{D}(\boldsymbol{w}), \mathbb{D}(\boldsymbol{f})-\mathbb{C}\rangle=\mathbb{D}(\boldsymbol{w}): \frac{d}{d t} \frac{\partial V}{\partial \mathbb{D}}
$$

С целью вьвода уравнения для $\rho, \boldsymbol{w}$ преобразуем правую часть (1.10) (с учетом (1.3), $(0.3),(0.1)$ и $(1.6))$ :

$$
\begin{aligned}
\mathbb{D}(\boldsymbol{w}): \frac{d}{d t}(\mathbb{P}+p \mathbb{I})= & \operatorname{div}\left(\frac{d \mathbb{P}}{d t} \boldsymbol{w}\right)-\boldsymbol{w} \cdot \frac{d}{d t}(\rho \boldsymbol{w})-\boldsymbol{w} \cdot[\mathbb{P}, \boldsymbol{u}]_{(1)}+\frac{d p}{d t} \operatorname{div} \boldsymbol{w} \\
= & \operatorname{div}\left(\frac{d \mathbb{P}}{d t} \boldsymbol{w}\right)-\rho \frac{d}{d t} \frac{|\boldsymbol{w}|^{2}}{2}+\rho|\boldsymbol{w}|^{2} \operatorname{div} \boldsymbol{u} \\
& -\boldsymbol{w} \cdot[\mathbb{P}, \boldsymbol{u}]_{(1)}-\rho p^{\prime}(\rho)(\operatorname{div} \boldsymbol{u}) \operatorname{div} \boldsymbol{w} .
\end{aligned}
$$

В итоге (1.10), сложенное с (0.1), умноженным на $|\boldsymbol{w}|^{2} / 2$, после интегрирования по $\Omega$ дает третье энергетическое тождество

$$
\begin{aligned}
\frac{d}{d t} \int_{\Omega} & \frac{\rho|\boldsymbol{w}|^{2}}{2} d \boldsymbol{x}+\int_{\Omega} \frac{\partial^{2} V}{\partial \mathbb{D}^{2}}\langle\mathbb{D}(\boldsymbol{w}), \mathbb{D}(\boldsymbol{w})\rangle d \boldsymbol{x} \\
= & \int_{\Omega} \rho|\boldsymbol{w}|^{2} \operatorname{div} \boldsymbol{u} \boldsymbol{x} \\
& +\int_{\Omega}\left(\frac{\partial^{2} V}{\partial \mathbb{D}^{2}}\langle\mathbb{D}(\boldsymbol{w}), \mathbb{D}(\boldsymbol{f})-\mathbb{C}\rangle-\boldsymbol{w} \cdot[\mathbb{P}, \boldsymbol{u}]_{(1)}-\rho p^{\prime}(\rho)(\operatorname{div} \boldsymbol{u}) \operatorname{div} \boldsymbol{w}\right) d \boldsymbol{x} \\
& +\int_{\partial \Omega}\left(\frac{d \mathbb{P}}{d t} \boldsymbol{w}-\frac{\rho|\boldsymbol{w}|^{2}}{2} \boldsymbol{u}\right) \cdot \boldsymbol{n} d S .
\end{aligned}
$$

В $(1.11)$ входят производные от $\rho$ (через $p$ в члене $\left.[\mathbb{P}, \boldsymbol{u}]_{(1)}\right)$, однако их можно убрать, воспользовавшись очевидным тождеством

$$
\int_{\Omega} \boldsymbol{w} \cdot[p \mathbb{I}, \boldsymbol{u}]_{(1)} d \boldsymbol{x}=-\int_{\Omega} p\left((\nabla \otimes \boldsymbol{u})^{*}:(\nabla \otimes \boldsymbol{w})+\boldsymbol{w} \cdot \nabla \operatorname{div} \boldsymbol{u}\right) d \boldsymbol{x}+\int_{\partial \Omega} p\left((\nabla \otimes \boldsymbol{u})^{*} \boldsymbol{w}\right) \cdot \boldsymbol{n} d S .
$$

Процесс вывода энергетических тождеств может быть продолжен и далее, но при этом более не удается избежать появления производных от $\rho$, что, впрочем, не мешает 
получению оценок, так как уже вторая энергетическая оценка (следствие (1.7) или (1.8)) заведомо позволяет оценить $\nabla \rho$ (см. п. 4). Продемонстрируем вьвод четвертого энергетического тождества. С этой целью сделаем еще одно дифференциальное продолжение уравнения импульса. Взяв $\partial / \partial x_{k}$ от (1.9) и применив к полученному уравнению операцию

$$
\frac{\partial^{2} V}{\partial \mathbb{D}^{2}}\left\langle\frac{\partial \mathbb{D}(\boldsymbol{w})}{\partial x_{k}}, \cdot\right\rangle
$$

получим равенство

$$
\frac{\partial^{2} V}{\partial \mathbb{D}^{2}}\left\langle\frac{\partial \mathbb{D}(\boldsymbol{w})}{\partial x_{k}}, \frac{\partial}{\partial x_{k}} \frac{d \mathbb{D}}{d t}\right\rangle=\frac{\partial^{2} V}{\partial \mathbb{D}^{2}}\left\langle\frac{\partial \mathbb{D}(\boldsymbol{w})}{\partial x_{k}}, \frac{\partial \mathbb{D}(\boldsymbol{w})}{\partial x_{k}}\right\rangle+\frac{\partial^{2} V}{\partial \mathbb{D}^{2}}\left\langle\frac{\partial \mathbb{D}(\boldsymbol{w})}{\partial x_{k}}, \frac{\partial \mathbb{D}(\boldsymbol{f})}{\partial x_{k}}-\frac{\partial \mathbb{C}}{\partial x_{k}}\right\rangle
$$

С другой стороны, имеем два представления одного и того же выражения: первое - следствие определения (0.6):

$$
\begin{aligned}
\frac{\partial \mathbb{D}(\boldsymbol{w})}{\partial x_{k}}: \frac{\partial}{\partial x_{k}} \frac{d \mathbb{P}}{d t}= & -\frac{\partial \operatorname{div} \boldsymbol{w}}{\partial x_{k}} \frac{\partial}{\partial x_{k}} \frac{d p}{d t}+\frac{\partial^{2} V}{\partial \mathbb{D}^{2}}\left\langle\frac{\partial \mathbb{D}(\boldsymbol{w})}{\partial x_{k}}, \frac{\partial}{\partial x_{k}} \frac{d \mathbb{D}}{d t}\right\rangle \\
& +\frac{\partial^{3} V}{\partial \mathbb{D}^{3}}\left\langle\frac{\partial \mathbb{D}(\boldsymbol{w})}{\partial x_{k}}, \frac{\partial \mathbb{D}}{\partial x_{k}}, \frac{d \mathbb{D}}{d t}\right\rangle
\end{aligned}
$$

а второе - следствие $(1.3),(0.1)$ и (0.3):

$$
\begin{aligned}
\mathbb{D}\left(\frac{\partial \boldsymbol{w}}{\partial x_{k}}\right): \frac{\partial}{\partial x_{k}} \frac{d \mathbb{P}}{d t}= & \operatorname{div}\left(\left(\frac{\partial}{\partial x_{k}} \frac{d \mathbb{P}}{d t}\right) \frac{\partial \boldsymbol{w}}{\partial x_{k}}\right)-\frac{\partial \boldsymbol{w}}{\partial x_{k}} \cdot \frac{\partial}{\partial x_{k}} \operatorname{div} \frac{d \mathbb{P}}{d t} \\
= & \operatorname{div}\left(\left(\frac{\partial}{\partial x_{k}} \frac{d \mathbb{P}}{d t}\right) \frac{\partial \boldsymbol{w}}{\partial x_{k}}\right)+\frac{\partial \boldsymbol{w}}{\partial x_{k}} \cdot\left(\boldsymbol{w} \operatorname{div} \boldsymbol{u} \frac{\partial \rho}{\partial x_{k}}+\rho \frac{\partial}{\partial x_{k}}(\boldsymbol{w} \operatorname{div} \boldsymbol{u})\right. \\
& \left.-\rho \frac{\partial}{\partial x_{k}} \frac{d \boldsymbol{w}}{d t}-\frac{\partial \rho}{\partial x_{k}} \frac{d \boldsymbol{w}}{d t}-\frac{\partial}{\partial x_{k}}[\mathbb{P}, \boldsymbol{u}]_{(1)}\right)
\end{aligned}
$$

Наконец, рассмотрим равенство (вытекающее из (1.1))

$$
\frac{d}{d t} \frac{\partial \boldsymbol{w}}{\partial x_{k}}=\frac{\partial}{\partial x_{k}} \frac{d \boldsymbol{w}}{d t}-\sum_{j=1}^{n} \frac{\partial u_{j}}{\partial x_{k}} \frac{\partial \boldsymbol{w}}{\partial x_{j}}
$$

умножив которое на $\rho\left(\partial \boldsymbol{w} / \partial x_{k}\right)$ и сложив с $(0.1)$, умноженным на $\left|\partial \boldsymbol{w} / \partial x_{k}\right|^{2} / 2$, выведем

$$
\frac{d}{d t}\left(\frac{\rho}{2}\left|\frac{\partial \boldsymbol{w}}{\partial x_{k}}\right|^{2}\right)+\frac{\rho}{2}\left|\frac{\partial \boldsymbol{w}}{\partial x_{k}}\right|^{2} \operatorname{div} \boldsymbol{u}=\rho \frac{\partial \boldsymbol{w}}{\partial x_{k}} \cdot \frac{\partial}{\partial x_{k}} \frac{d \boldsymbol{w}}{d t}-\rho \sum_{j=1}^{n} \frac{\partial \boldsymbol{w}}{\partial x_{j}} \cdot \frac{\partial \boldsymbol{w}}{\partial x_{k}} \frac{\partial u_{j}}{\partial x_{k}}
$$

что после интегрирования по $\Omega$ с учетом $(1.12)-(1.14),(1.6)$ и (1.9) дает четвертое энергетическое тождество

$$
\frac{d}{d t} \int_{\Omega} \frac{\rho|\nabla \otimes \boldsymbol{w}|^{2}}{2} d \boldsymbol{x}+\sum_{k=1}^{n} \int_{\Omega} \frac{\partial^{2} V}{\partial \mathbb{D}^{2}}\left\langle\frac{\partial \mathbb{D}(\boldsymbol{w})}{\partial x_{k}}, \frac{\partial \mathbb{D}(\boldsymbol{w})}{\partial x_{k}}\right\rangle d \boldsymbol{x}
$$




$$
\begin{aligned}
&= \sum_{k=1}^{n} \int_{\Omega}\left(\frac{\partial^{2} V}{\partial \mathbb{D}^{2}}\left\langle\frac{\partial \mathbb{D}(\boldsymbol{w})}{\partial x_{k}}, \frac{\partial \mathbb{C}}{\partial x_{k}}-\frac{\partial \mathbb{D}(\boldsymbol{f})}{\partial x_{k}}\right\rangle\right. \\
&\left.+\frac{\partial^{3} V}{\partial \mathbb{D}^{3}}\left\langle\frac{\partial \mathbb{D}(\boldsymbol{w})}{\partial x_{k}}, \frac{\partial \mathbb{D}}{\partial x_{k}}, \mathbb{C}-\mathbb{D}(\boldsymbol{w})-\mathbb{D}(\boldsymbol{f})\right\rangle\right) d \boldsymbol{x} \\
&+\sum_{k=1}^{n} \int_{\Omega}\left(\frac{\partial \rho}{\partial x_{k}} \frac{\partial \boldsymbol{w}}{\partial x_{k}} \cdot\left(\boldsymbol{w} \operatorname{div} \boldsymbol{u}-\frac{d \boldsymbol{w}}{d t}\right)-\frac{\partial \operatorname{div} \boldsymbol{w}}{\partial x_{k}} \frac{\partial}{\partial x_{k}}\left(\rho p^{\prime}(\rho) \operatorname{div} \boldsymbol{u}\right)\right) d \boldsymbol{x} \\
&+\int_{\Omega}\left(\rho(\nabla \otimes \boldsymbol{w}):(\nabla \otimes(\boldsymbol{w} \operatorname{div} \boldsymbol{u}))-(\nabla \otimes \boldsymbol{w}):\left(\nabla \otimes[\mathbb{P}, \boldsymbol{u}]_{(1)}\right)\right. \\
&\left.-\rho \sum_{j, k=1}^{n} \frac{\partial \boldsymbol{w}}{\partial x_{j}} \cdot \frac{\partial \boldsymbol{w}}{\partial x_{k}} \frac{\partial u_{j}}{\partial x_{k}}\right) d \boldsymbol{x}+\int_{\partial \Omega}\left(\sum_{k=1}^{n}\left(\frac{\partial}{\partial x_{k}} \frac{d \mathbb{P}}{d t}\right) \frac{\partial \boldsymbol{w}}{\partial x_{k}}-\frac{\rho|\nabla \otimes \boldsymbol{w}|^{2}}{2} \boldsymbol{u}\right) \cdot \boldsymbol{n} d S . \\
&(1.15)
\end{aligned}
$$

2. Оценки для нелинейной эллиптической системы (0.3). Энергетические тождества $(1.5),(1.7),(1.8),(1.11)$ и (1.15) содержат в правой части нелинейные выражения от производных от $\boldsymbol{u}$, а в левой части положительные (в случае знакоопределенности квадратичной формы $\partial^{2} V / \partial \mathbb{D}^{2}$ ) слагаемые, позволяющие оценить $\boldsymbol{w}$. В связи с этим вьвод оценок из этих тождеств сводится к умению получать оценки решений $(0.3)$ как эллиптической системы для $\boldsymbol{u}$. Этому вопросу и посвящен данный пункт. Здесь мы также уточним класс рассматриваемых нами потенщиалов $V$ и изучим их свойства.

Итак, рассмотрим нелинейную эллиптическую систему

$$
\operatorname{div} \frac{\partial V(\mathbb{D}(\boldsymbol{u}))}{\partial \mathbb{D}}=\mathbf{F}
$$

и получим для нее энергетические тождества, а затем оценки. Снова предполагаем, что $\Omega$ - область в $\mathbb{R}^{n}$ с гладкой гранищей или параллелепипед и в $\Omega$ имеется достаточно гладкое решение $\boldsymbol{u}$ системы (2.1). Начнем с абстрактной схемы.

Из (2.1) следует равенство (напомним, что мы предполагаем симметричность тензоpa $\partial V / \partial \mathbb{D})$

$$
\mathbf{F} \cdot \boldsymbol{u}=\operatorname{div}\left[\frac{\partial V}{\partial \mathbb{D}}, \boldsymbol{u}\right]^{(0)}-\frac{\partial V}{\partial \mathbb{D}}: \mathbb{D}
$$

проинтегрировав которое по $\Omega$, получим первое энергетическое тождество

$$
\int_{\Omega} \frac{\partial V}{\partial \mathbb{D}}: \mathbb{D} d \boldsymbol{x}=-\int_{\Omega} \mathbf{F} \cdot \boldsymbol{u} d \boldsymbol{x}+\int_{\partial \Omega}\left[\frac{\partial V}{\partial \mathbb{D}}, \boldsymbol{u}\right]^{(0)} \cdot \boldsymbol{n} d S
$$

Далее, с помощью (2.1) преобразуем выражение

$$
\begin{aligned}
\sum_{i, k=1}^{n} \frac{\partial F_{i}}{\partial x_{k}} \frac{\partial u_{i}}{\partial x_{k}} & =\sum_{i, j, k=1}^{n} \frac{\partial}{\partial x_{j}}\left(\frac{\partial}{\partial x_{k}}\left(\frac{\partial V}{\partial \mathbb{D}}\right)_{i j} \frac{\partial u_{i}}{\partial x_{k}}\right)-\sum_{i, j, k=1}^{n} \frac{\partial}{\partial x_{k}}\left(\frac{\partial V}{\partial \mathbb{D}}\right)_{i j} \frac{\partial D_{i j}}{\partial x_{k}} \\
& =\operatorname{div}\left[\frac{\partial V}{\partial \mathbb{D}}, \boldsymbol{u}\right]^{(1)}-\sum_{k=1}^{n} \frac{\partial^{2} V}{\partial \mathbb{D}^{2}}\left\langle\frac{\partial \mathbb{D}}{\partial x_{k}}, \frac{\partial \mathbb{D}}{\partial x_{k}}\right\rangle,
\end{aligned}
$$


что после интегрирования по $\Omega$ дает второе энергетическое тождество

$$
\sum_{k=1}^{n} \int_{\Omega} \frac{\partial^{2} V}{\partial \mathbb{D}^{2}}\left\langle\frac{\partial \mathbb{D}}{\partial x_{k}}, \frac{\partial \mathbb{D}}{\partial x_{k}}\right\rangle d \boldsymbol{x}=\int_{\Omega} \mathbf{F} \cdot \Delta \boldsymbol{u} d \boldsymbol{x}+\int_{\partial \Omega}\left(\left[\frac{\partial V}{\partial \mathbb{D}}, \boldsymbol{u}\right]^{(1)}-(\nabla \otimes \boldsymbol{u}) \mathbf{F}\right) \cdot \boldsymbol{n} d S
$$

Этот процесс может быть продолжен и далее. Продемонстрируем получение третьего энергетического тождества. Преобразуем выражение

$$
\begin{aligned}
& \sum_{i, j, k=1}^{n} \frac{\partial^{2} F_{i}}{\partial x_{j} \partial x_{k}} \frac{\partial^{2} u_{i}}{\partial x_{j} \partial x_{k}}=\sum_{i, j, k, l=1}^{n} \frac{\partial}{\partial x_{l}}\left(\frac{\partial^{2}}{\partial x_{j} \partial x_{k}}\left(\frac{\partial V}{\partial \mathbb{D}}\right)_{i l} \frac{\partial^{2} u_{i}}{\partial x_{j} \partial x_{k}}\right) \\
& \quad-\sum_{i, j, k, l, p, q=1}^{n} \frac{\partial^{3} u_{i}}{\partial x_{j} \partial x_{k} \partial x_{l}} \frac{\partial}{\partial x_{j}}\left(\frac{\partial^{2} V}{\partial D_{i l} \partial D_{p q}} \frac{\partial D_{p q}}{\partial x_{k}}\right) \\
& =\operatorname{div}\left[\frac{\partial V}{\partial \mathbb{D}}, \boldsymbol{u}\right]^{(2)}-\sum_{j, k=1}^{n} \frac{\partial^{2} V}{\partial \mathbb{D}^{2}}\left\langle\frac{\partial^{2} \mathbb{D}}{\partial x_{j} \partial x_{k}}, \frac{\partial^{2} \mathbb{D}}{\partial x_{j} \partial x_{k}}\right\rangle-\sum_{j, k=1}^{n} \frac{\partial^{3} V}{\partial \mathbb{D}^{3}}\left\langle\frac{\partial^{2} \mathbb{D}}{\partial x_{j} \partial x_{k}}, \frac{\partial \mathbb{D}}{\partial x_{j}}, \frac{\partial \mathbb{D}}{\partial x_{k}}\right\rangle .
\end{aligned}
$$

После интегрирования по $\Omega$ выведем требуемое тождество

$$
\begin{aligned}
& \sum_{j, k=1}^{n} \int_{\Omega} \frac{\partial^{2} V}{\partial \mathbb{D}^{2}}\left\langle\frac{\partial^{2} \mathbb{D}}{\partial x_{j} \partial x_{k}}, \frac{\partial^{2} \mathbb{D}}{\partial x_{j} \partial x_{k}}\right\rangle d \boldsymbol{x}=-\sum_{j, k=1}^{n} \int_{\Omega} \frac{\partial^{3} V}{\partial \mathbb{D}^{3}}\left\langle\frac{\partial^{2} \mathbb{D}}{\partial x_{j} \partial x_{k}}, \frac{\partial \mathbb{D}}{\partial x_{j}}, \frac{\partial \mathbb{D}}{\partial x_{k}}\right\rangle d \boldsymbol{x} \\
& \quad+\int_{\Omega}(\nabla \otimes \mathbf{F}):(\nabla \otimes \Delta \boldsymbol{u}) d \boldsymbol{x}+\int_{\partial \Omega}\left(\left[\frac{\partial V}{\partial \mathbb{D}}, \boldsymbol{u}\right]^{(2)}-\sum_{j=1}^{n}\left(\nabla \otimes \frac{\partial \boldsymbol{u}}{\partial x_{j}}\right) \frac{\partial \mathbf{F}}{\partial x_{j}}\right) \cdot \boldsymbol{n} d S
\end{aligned}
$$

Для того чтобы соотношения (2.2)-(2.4) давали оценки, нужна, как минимум, положительность квадратичной формы

$$
\frac{\partial^{2} V}{\partial \mathbb{D}^{2}}\langle\mathbb{A}, \mathbb{A}\rangle \geqslant 0
$$

Если она имеет место, то тензор $\partial V / \partial \mathbb{D}$ будет положительным и монотонным, т.е.

$$
\frac{\partial V}{\partial \mathbb{D}}: \mathbb{D} \geqslant 0, \quad\left(\frac{\partial V}{\partial \mathbb{D}}\left(\mathbb{D}_{1}\right)-\frac{\partial V}{\partial \mathbb{D}}\left(\mathbb{D}_{2}\right)\right):\left(\mathbb{D}_{1}-\mathbb{D}_{2}\right) \geqslant 0
$$

при дополнительных условиях

$$
V(0)=0, \quad \frac{\partial V}{\partial \mathbb{D}}(0)=0
$$

(доказательство см., например, в [4]), заведомо вьполненных для потенциалов $V$, имеющих смысл в механике жидкости (в силу аксиом Стокса). В [4] выделен некоторый класс потенциалов $V$, в котором имеют место свойства (2.5) (и даже более сильные свойства коэрцитивности и строгой монотонности), но он по существу сводится к квадратичным: $V=G\left(|\mathbb{D}|^{2}\right)$. Мы же будем рассматривать потенциалы вида

$$
V(\mathbb{D})=\Lambda\left((\operatorname{tr} \mathbb{D})^{2}\right)+\sum_{s=1}^{N} \Gamma_{s}\left(\left|\mathbb{D}^{s}\right|^{2}\right)
$$


(где $N$ - произвольное натуральное число) с неотрицательными монотонными вьпуклыми функциями $\Lambda$ и $\Gamma_{s}$, получим для них энергетические (п. 2) и другие (п. 4) оценки системы (2.1), а также оценки соответствующих эволюционных уравнений (0.1)-(0.4) (п. 3,4$)$.

Прежде всего необходимо найти производные $V$. Непосредственным вычислением проверяется формула

$$
\frac{\partial \operatorname{tr}\left(\mathbb{D}^{m} \mathbb{A}\right)}{\partial \mathbb{D}}: \mathbb{B}=m \operatorname{tr}\left(\mathbb{D}^{m-1} \mathbb{A} \mathbb{B}\right)
$$

(справедливая для всех натуральных $m$ и симметричных тензоров $\mathbb{A}, \mathbb{B}, \mathbb{D})$, с помощью которой нетрудно последовательно выписать требуемые представления (в которых $\mathbb{A}, \mathbb{B}$ и $\mathbb{C}$ - постоянные тензоры):

$$
\begin{aligned}
& \frac{\partial V}{\partial \mathbb{D}}: \mathbb{A}=2 \Lambda^{\prime}\left((\operatorname{tr} \mathbb{D})^{2}\right)(\operatorname{tr} \mathbb{D})(\operatorname{tr} \mathbb{A})+\sum_{s=1}^{N} 2 s \Gamma_{s}^{\prime}\left(\left|\mathbb{D}^{s}\right|^{2}\right) \operatorname{tr}\left(\mathbb{D}^{2 s-1} \mathbb{A}\right) \\
& \frac{\partial^{2} V}{\partial \mathbb{D}^{2}}\langle\mathbb{A}, \mathbb{B}\rangle=\sum_{k, l=1}^{n} B_{k l} \frac{\partial}{\partial D_{k l}}\left(\frac{\partial V}{\partial \mathbb{D}}: \mathbb{A}\right) \\
&=2\left(\Lambda^{\prime}\left((\operatorname{tr} \mathbb{D})^{2}\right)+2\left(\operatorname{tr} \mathbb{D}^{2} \Lambda^{\prime \prime}\left((\operatorname{tr} \mathbb{D})^{2}\right)\right)(\operatorname{tr} \mathbb{A})(\operatorname{tr} \mathbb{B})\right. \\
& \quad+\sum_{s=1}^{N}\left(4 s^{2} \Gamma_{s}^{\prime \prime}\left(\left|\mathbb{D}^{s}\right|^{2}\right) \operatorname{tr}\left(\mathbb{D}^{2 s-1} \mathbb{A}\right) \operatorname{tr}\left(\mathbb{D}^{2 s-1} \mathbb{B}\right)+2 s(2 s-1) \Gamma_{s}^{\prime}\left(\left|\mathbb{D}^{s}\right|^{2}\right) \operatorname{tr}\left(\mathbb{D}^{2 s-2} \mathbb{A}\right)\right) \\
& \frac{\partial^{3} V}{\partial \mathbb{D}^{3}}\left\langle\mathbb{A}, \mathbb{B}^{2}, \mathbb{C}\right\rangle=\sum_{p, q=1}^{n} C_{p q} \frac{\partial}{\partial D_{p q}} \frac{\partial^{2} V}{\partial \mathbb{D}^{2}}\langle\mathbb{A}, \mathbb{B}\rangle \\
&=4\left(3 \Lambda ^ { \prime \prime } \left(\left(\operatorname{tr} \mathbb{D}^{2}\right)+2\left(\operatorname{tr} \mathbb{D}^{2} \Lambda^{\prime \prime \prime}\left((\operatorname{tr} \mathbb{D})^{2}\right)\right)(\operatorname{tr} \mathbb{D})(\operatorname{tr} \mathbb{A})\left(\operatorname{tr} \mathbb{B}^{2}\right)(\operatorname{tr} \mathbb{C})\right.\right. \\
& \quad+\sum_{s=1}^{N}\left(8 s^{3} \Gamma_{s}^{\prime \prime \prime}\left(\left|\mathbb{D}^{s}\right|^{2}\right) \operatorname{tr}\left(\mathbb{D}^{2 s-1} \mathbb{A}\right) \operatorname{tr}\left(\mathbb{D}^{2 s-1} \mathbb{B}\right) \operatorname{tr}\left(\mathbb{D}^{2 s-1} \mathbb{C}\right)\right. \\
& \quad+4 s^{2}(2 s-1) \Gamma_{s}^{\prime \prime}\left(\left|\mathbb{D}^{s}\right|^{2}\right)\left(\operatorname{tr}\left(\mathbb{D}^{2 s-2} \mathbb{A} \mathbb{B}\right) \operatorname{tr}\left(\mathbb{D}^{2 s-1} \mathbb{C}\right)\right. \\
& \quad+\operatorname{tr}\left(\mathbb{D}^{2 s-2} \mathbb{A} \mathbb{C}\right) \operatorname{tr}\left(\mathbb{D}^{2 s-1} \mathbb{B}^{2 s}+\operatorname{tr}\left(\mathbb{D}^{2 s-2} \mathbb{B} \mathbb{C}\right) \operatorname{tr}\left(\mathbb{D}^{2 s-1} \mathbb{A}\right)\right) \\
&\left.\quad+2 s(2 s-1)(2 s-2) \Gamma_{s}^{\prime}\left(\left|\mathbb{D}^{s}\right|^{2}\right) \operatorname{tr}\left(\mathbb{D}^{2 s-3} \mathbb{A B} \mathbb{C}\right)\right)
\end{aligned}
$$

(последнее слагаемое в (2.9) при $s=1$ по определению полагаем нулем).

Отметим некоторые свойства $V$, вытекающие из (2.7)-(2.9). Из (2.8) вытекает неравенство Коши для формы $\partial^{2} V / \partial \mathbb{D}^{2}$ :

$$
2 \frac{\partial^{2} V}{\partial \mathbb{D}^{2}}\langle\mathbb{A}, \mathbb{B}\rangle \leqslant \frac{\partial^{2} V}{\partial \mathbb{D}^{2}}\langle\mathbb{A}, \mathbb{A}\rangle+\frac{\partial^{2} V}{\partial \mathbb{D}^{2}}\langle\mathbb{B}, \mathbb{B}\rangle
$$

а также оценка для ее нормы:

$$
\frac{\partial^{2} V}{\partial \mathbb{D}^{2}}\langle\mathbb{A}, \mathbb{A}\rangle \leqslant \Phi^{2}(\mathbb{D})|\mathbb{A}|^{2}
$$


где

$$
\begin{aligned}
\Phi(\mathbb{D})= & \sqrt{2 n \Lambda^{\prime}\left((\operatorname{tr} \mathbb{D})^{2}\right)}+2|\operatorname{tr} \mathbb{D}| \sqrt{n \Lambda^{\prime \prime}\left((\operatorname{tr} \mathbb{D})^{2}\right)} \\
& +\sum_{s=1}^{N}\left(\left|\mathbb{D}^{s-1}\right| \sqrt{2 s(2 s-1) \Gamma_{s}^{\prime}\left(\left|\mathbb{D}^{s}\right|^{2}\right)}+2 s\left|\mathbb{D}^{2 s-1}\right| \sqrt{\Gamma_{s}^{\prime \prime}\left(\left|\mathbb{D}^{s}\right|^{2}\right)}\right)
\end{aligned}
$$

Обозначив через $\Psi(\mathbb{D})$ левую часть $(2.3)$, мы можем записать следующую оценку всех производных $\partial V / \partial \mathbb{D}$ :

$$
\left|\nabla \wedge \frac{\partial V}{\partial \mathbb{D}}\right|^{2} \leqslant(2 N+2) \Phi^{2}(\mathbb{D}) \Psi(\mathbb{D}) .
$$

Действительно, непосредственньм вычислением из (2.7) вьводится соотношение

$$
\begin{aligned}
\left|\frac{\partial}{\partial x_{k}} \frac{\partial V}{\partial \mathbb{D}}\right|^{2} \leqslant & (2 N+2)\left(4 n \Lambda^{\prime 2}\left((\operatorname{tr} \mathbb{D})^{2}\right)\left(\frac{\partial \operatorname{tr} \mathbb{D}}{\partial x_{k}}\right)^{2}+16 n(\operatorname{tr} \mathbb{D})^{4} \Lambda^{\prime \prime 2}\left((\operatorname{tr} \mathbb{D})^{2}\right)\left(\frac{\partial \operatorname{tr} \mathbb{D}}{\partial x_{k}}\right)^{2}\right. \\
& +\sum_{s=1}^{N}\left(4 s^{2} \Gamma_{s}^{\prime 2}\left(\left|\mathbb{D}^{s}\right|^{2}\right)(2 s-1)^{2}\left|\mathbb{D}^{s-1} \mathbb{D}^{s-1} \frac{\partial \mathbb{D}}{\partial x_{k}}\right|^{2}\right. \\
& \left.\left.+16 s^{4} \Gamma_{s}^{\prime \prime 2}\left(\left|\mathbb{D}^{s}\right|^{2}\right)\left|\mathbb{D}^{2 s-1}\right|^{2}\left(\operatorname{tr} \mathbb{D}^{2 s-1} \frac{\partial \mathbb{D}}{\partial x_{k}}\right)^{2}\right)\right)
\end{aligned}
$$

которое влечет (2.13) после применения элементарных неравенств.

Чтобы избавиться от граничных интегралов в (2.2)-(2.4), рассмотрим для (2.1) краевую задачу в параллелепипеде $\Omega$ с краевьми условиями периодичности (аналогично (3.5)). Выпишем тождества $(2.2),(2.3)$ для потенциала (2.6). Равенство (2.2) примет вид

$$
\int_{\Omega}\left(2 \Lambda^{\prime}\left((\operatorname{tr} \mathbb{D})^{2}\right)(\operatorname{tr} \mathbb{D})^{2}+\sum_{s=1}^{N} 2 s \Gamma_{s}^{\prime}\left(\left|\mathbb{D}^{s}\right|^{2}\right)\left|\mathbb{D}^{s}\right|^{2}\right) d \boldsymbol{x}=-\int_{\Omega} \mathbf{F} \cdot \boldsymbol{u} d \boldsymbol{x} .
$$

Введя обозначения

$$
\varkappa(\xi)=\int_{0}^{\xi} \sqrt{\Lambda^{\prime \prime}(\eta)} d \eta+1, \quad \nu_{s}(\xi)=\int_{0}^{\xi} \sqrt{\Gamma_{s}^{\prime \prime}(\eta)} d \eta+1
$$

запишем (2.3) в виде

$$
\int_{\Omega} \Psi(\mathbb{D}) d \boldsymbol{x}=\int_{\Omega} \mathbf{F} \cdot \Delta \boldsymbol{u} d \boldsymbol{x}
$$

c

$$
\begin{aligned}
\Psi(\mathbb{D})= & 2 \Lambda^{\prime}\left((\operatorname{tr} \mathbb{D})^{2}\right)|\nabla \operatorname{tr} \mathbb{D}|^{2}+\left|\nabla \varkappa\left((\operatorname{tr} \mathbb{D})^{2}\right)\right|^{2} \\
& +\sum_{s=1}^{N}\left(2 s(2 s-1) \Gamma_{s}^{\prime}\left(\left|\mathbb{D}^{s}\right|^{2}\right) \sum_{k=1}^{n}\left|\mathbb{D}^{s-1} \frac{\partial \mathbb{D}}{\partial x_{k}}\right|^{2}+\left|\nabla \nu_{s}\left(\left|\mathbb{D}^{s}\right|^{2}\right)\right|^{2}\right) .
\end{aligned}
$$

Если ограничиться случаем, когда в (2.6) верно

$$
\Gamma_{1}^{\prime}(\xi) \geqslant C_{1}>0
$$


то форма $\partial^{2} V / \partial \mathbb{D}^{2}$ положительно определена:

$$
\frac{\partial^{2} V}{\partial \mathbb{D}^{2}}\langle\mathbb{A}, \mathbb{A}\rangle \geqslant 2 C_{1} \mid \mathbb{A}^{2}
$$

и, в частности, $\Psi(\mathbb{D}) \geqslant 2 C_{1}|\nabla \wedge \mathbb{D}|^{2}$. Тогда из $(2.14),(2.16)$ вытекает вторая энергетическая оценка

$$
\int_{\Omega} \Psi(\mathbb{D}) d \boldsymbol{x} \leqslant C_{2}\left(C_{1}, \Omega\right)\|\mathbf{F}\|_{L_{2}(\Omega)}^{2}
$$

3. Энергетические оценки в модели Бюргерса с быстро растущим потенциалом напряжений. В этом пункте мы покажем, как энергетические тождества, выведенные в п. 1, могут быть превращены в оценки решений (0.1)-(0.4) на примере модели Бюргерса (т.е. в (0.6) $p \equiv 0)$ в случае $n=2,3$ с $V$ в форме (2.6), где коэффициенты $\Lambda$ и $\Gamma_{s}$ вьпуклы, монотонны (что гарантирует осмысленность обозначений $(2.15)$ ), удовлетворяют (2.18) и обладают следующими свойствами:

$$
\begin{array}{cc}
\exists s=2^{m}, m \in \mathbb{N}_{0}: \quad \Gamma_{s}^{\prime}(\xi) \rightarrow+\infty \text { при } \xi \rightarrow M<\infty, \\
\Lambda^{\prime}(\xi)+\Lambda^{\prime \prime}(\xi) \leqslant C_{3}\left(1+\Lambda^{\alpha}(\xi)\right), & \Gamma_{s}^{\prime}(\xi)+\Gamma_{s}^{\prime \prime}(\xi) \leqslant C_{3}\left(1+\Gamma_{s}^{\alpha}(\xi)\right), \\
\Lambda^{\prime}(\xi)+\Lambda^{\prime \prime}(\xi) \leqslant C_{3}\left(1+\varkappa^{\beta}(\xi)\right), & \Gamma_{s}^{\prime}(\xi)+\Gamma_{s}^{\prime \prime}(\xi) \leqslant C_{3}\left(1+\nu_{s}^{\beta}(\xi)\right),
\end{array}
$$

причем постоянные $\alpha$ и $\beta$ удовлетворяют ограничениям

$$
\alpha>1, \quad \beta>2, \quad \frac{\beta-2}{\beta} \alpha<\frac{4-n}{n}
$$

(отметим, что первые два неравенства в (3.4) необходимы для (3.1)). Перечисленньм требованиям удовлетворяют, например, функции

$$
\Lambda(\xi)=\Gamma_{s}(\xi)=(1-\xi)^{-\gamma}, \quad \gamma>\frac{2 n}{4-n} .
$$

Рассмотрим для $(0.1)-(0.4),(0.6)$ начально-краевую задачу в цилиндре $Q_{T}=\Omega \times$ $(0, T)$, где $\Omega=\prod_{i=1}^{n}\left(0, \lambda_{i}\right)$, с краевыми условиями периодичности

$$
\rho, \boldsymbol{u} \text { периодичны по } x_{i} \text { с периодами } \lambda_{i}
$$

и начальными данными

$$
\left.\rho\right|_{t=0}=\rho_{0}>0,\left.\quad u\right|_{t=0}=u_{0}
$$

(отметим, однако, что в тождествах $(1.5),(1.7),(1.8),(1.11)$ граничные интегралы исчезают и для задачи с краевым условием прилипания $\left.\boldsymbol{u}\right|_{\partial \Omega}=0$ вместо (3.5) при дополнительном требовании $\left.\left.\boldsymbol{f}\right|_{\partial \Omega}=0\right)$. В этом пункте на основе энергетических тождеств из п. 1 и оценок из п. 2 мы получим оценки гладкого решения поставленной задачи (подразумеваем положительность плотности: $\rho>0)$.

Из (1.5) и тождества $\|\rho\|_{L_{1}(\Omega)}=$ const (следствия (0.1)) вытекает первая энергетическая оценка

$$
\int_{\Omega} \frac{\partial V}{\partial \mathbb{D}}: \mathbb{D} d \boldsymbol{x} d t \leqslant C_{4}\left(T,\left\|\rho_{0}\right\|_{L_{1}(\Omega)},\left\|\rho_{0}\left|\boldsymbol{u}_{0}\right|^{2}\right\|_{L_{1}(\Omega)},\|\boldsymbol{f}\|_{L_{1}\left(0, T, L_{\infty}(\Omega)\right)}\right) .
$$


Поскольку для всякого симметричного тензора $|\mathbb{B}|^{2}=\operatorname{tr} \mathbb{B}^{2} \leqslant \sqrt{n}\left|\mathbb{B}^{2}\right|$, из (3.7), (3.1) и (2.7) следует оценка

$$
|\mathbb{D}| \leqslant M_{1} \equiv \sqrt{n} M^{2^{-m}}
$$

на основе которой из (0.1) вьводим

$$
0<C_{5}\left(T, \inf \rho_{0}, M_{1}\right) \leqslant \rho \leqslant C_{6}\left(T, \sup \rho_{0}, M_{1}\right)<\infty
$$

Опираясь на (3.8) и (3.9), из (1.8) легко получим вторую энергетическую оценку

$$
\begin{gathered}
\sup _{0<t<T}\|V\|_{L_{1}(\Omega)} \leqslant C_{7}\left(T, C_{6}, \Omega,\left\|\left.V\right|_{t=0}\right\|_{L_{1}(\Omega)},\|f\|_{L_{2}\left(Q_{T}\right)}\right) \\
\|\boldsymbol{w}\|_{L_{2}\left(Q_{T}\right)} \leqslant C_{8}\left(T, C_{5}, C_{6}, \Omega,\left\|\left.V\right|_{t=0}\right\|_{L_{1}(\Omega)},\|\boldsymbol{f}\|_{L_{2}\left(Q_{T}\right)}\right) .
\end{gathered}
$$

Тождество (1.11) после применения неравенств $(3.8),(2.10),(2.19),(2.11)$ и (2.13) приводит к соотношению

$$
\begin{aligned}
& \frac{1}{2} \frac{d}{d t} \int_{\Omega} \rho|\boldsymbol{w}|^{2} d \boldsymbol{x}+C_{1}\|\mathbb{D}(\boldsymbol{w})\|_{L_{2}(\Omega)}^{2} \leqslant M_{1} \int_{\Omega} \rho|\boldsymbol{w}|^{2} d \boldsymbol{x} \\
& \quad+\int_{\Omega}\left(\Phi^{2}(\mathbb{D})\left(|\mathbb{D}(\boldsymbol{f})|^{2}+|\nabla \otimes \boldsymbol{u}|^{4}\right)+\sqrt{2 N+2}|\nabla \otimes \boldsymbol{u}| \cdot|\boldsymbol{w}| \cdot \Phi(\mathbb{D}) \sqrt{\Psi(\mathbb{D})}\right) d \boldsymbol{x} .
\end{aligned}
$$

Оценим слагаемые в его правой части. Введем обозначение

$$
q=\frac{2}{1+(\beta-2) \alpha / \beta}
$$

и отметим, что $q>n / 2$ ввиду (3.4). Из (3.2), (3.3) имеем оценку

$$
\begin{aligned}
\Phi^{2 q}(\mathbb{D}) \leqslant & C_{9}\left(C_{3}, n, N, M\right)\left(1+\Lambda^{(\beta-2) \alpha q / \beta}\left((\operatorname{tr} \mathbb{D})^{2}\right)+\sum_{s=1}^{N} \Gamma_{s}^{(\beta-2) \alpha q / \beta}\left(\left|\mathbb{D}^{s}\right|^{2}\right)\right) \\
& \times\left(1+\varkappa^{2 q}\left((\operatorname{tr} \mathbb{D})^{2}\right)+\sum_{s=1}^{N} \nu_{s}^{2 q}\left(\left|\mathbb{D}^{s}\right|^{2}\right)\right),
\end{aligned}
$$

которая после применения неравенства Гёльдера дает

$$
\begin{aligned}
& \|\Phi(\mathbb{D})\|_{L_{2 q}(\Omega)} \leqslant C_{10}\left(C_{9}, N\right)\left(1+\|V\|_{L_{1}(\Omega)}^{(\beta-2) \alpha /(2 \beta)}\right) \\
& \quad \times\left(1+\left\|\varkappa\left((\operatorname{tr} \mathbb{D})^{2}\right)\right\|_{L_{2 \beta q /(\beta-(\beta-2) \alpha q)}(\Omega)}+\sum_{s=1}^{N}\left\|\nu_{s}\left(\left|\mathbb{D}^{s}\right|^{2}\right)\right\|_{L_{2 \beta q /(\beta-(\beta-2) \alpha q)}(\Omega)}\right) .
\end{aligned}
$$

Поскольку $W_{2}^{1}(\Omega) \hookrightarrow L_{2 \beta q /(\beta-(\beta-2) \alpha q)}(\Omega)$, учитывая $(3.10),(3.13),(2.20)$ и $(2.17)$, мы можем записать

$$
\|\Phi(\mathbb{D})\|_{L_{2 q}(\Omega)} \leqslant C_{11}\left(C_{10}, C_{6}, C_{7}\right)\left(\|\boldsymbol{w}\|_{L_{2}(\Omega)}+1\right)
$$


а также

$$
\|\sqrt{\Psi(\mathbb{D})}\|_{L_{2}(\Omega)} \leqslant C_{2} C_{6}\|\boldsymbol{w}\|_{L_{2}(\Omega)}
$$

Теперь, применяя во втором интеграле в правой части (3.12) неравенство Гёльдера (для второго слагаемого - с показателями $4 n q /(2 q-n), 4 n q /(2 q(n-1)-n), 2 q$ и 2 ) и учитьвая вложение $W_{2}^{1}(\Omega) \hookrightarrow L_{4 n q /(2 q(n-1)-n)}(\Omega)$, мы можем оценить его величиной

$$
\begin{aligned}
& C_{11}^{2}\left(\|\boldsymbol{w}\|_{L_{2}(\Omega)}^{2}+1\right)\left(\|\nabla \otimes \boldsymbol{u}\|_{L_{4 q /(q-1)}(\Omega)}^{4}+\|\mathbb{D}(\boldsymbol{f})\|_{L_{2 q /(q-1)}(\Omega)}^{2}\right) \\
& \quad+C_{2} C_{6} C_{11} \sqrt{2 N+2}\left(\|\boldsymbol{w}\|_{L_{2}(\Omega)}^{2}+1\right)\|\mathbb{D}(\boldsymbol{w})\|_{L_{2}(\Omega)} \cdot\|\nabla \otimes \boldsymbol{u}\|_{L_{4 n q /(2 q-n)}(\Omega)}
\end{aligned}
$$

после чего неравенство (3.12) “замыкается” ввиду уже имеющихся оценок $(3.11),(3.8)$ и (3.9) и дает третью энергетическую оценку

$$
\begin{gathered}
\|\boldsymbol{w}\|_{L_{\infty}\left(0, T, L_{2}(\Omega)\right)} \leqslant C_{12}\left(T, C_{2}, C_{6}, C_{11}, C_{5},\left\|\left.\boldsymbol{w}\right|_{t=0}\right\|_{L_{2}(\Omega)},\|\boldsymbol{f}\|_{L_{2}\left(0, T, W_{2 q /(q-1)}^{1}(\Omega)\right)}\right) \\
\|\mathbb{D}(\boldsymbol{w})\|_{L_{2}\left(Q_{T}\right)} \leqslant C_{13}\left(T, C_{2}, C_{6}, C_{11}, C_{5},\left\|\left.\boldsymbol{w}\right|_{t=0}\right\|_{L_{2}(\Omega)},\|\boldsymbol{f}\|_{L_{2}\left(0, T, W_{2 q /(q-1)}^{1}(\Omega)\right)}\right)
\end{gathered}
$$

4. Дополнительные замечания. Дальнейшие оценки. Всякий шаг по вьводу очередной энергетической оценки эволюционной задачи требует соответствующей оценки стационарной системы (0.3). Так, для превращения (1.15) в оценку нужно сделать то же для (2.4), и т.д. Последнее, однако, представляет собой сложную проблему. До сих пор это удалось сделать (см. [4], [8] и ссылки там) лишь в случае $n=2$ для весьма специальных потенциалов (а именно, вида (2.6) с $N=1, \Gamma_{1}$, растущей как степенная функция с ограничениями сверху на показатель, и с ограничением $\operatorname{tr} \mathbb{D}=0$-задача типа Стокса).

Мы можем, тем не менее, сформулировать еще несколько оценок задачи (0.1)-(0.4), $(3.5),(3.6)$ (с $\mathbb{P}$, описанным в начале п. 3) в той мере, в которой это не требует дальнейшего (по сравнению с (2.20)) исследования системы (2.1):

$$
\begin{aligned}
\|\rho\|_{L_{\infty}\left(0, T, W_{2}^{1}(\Omega)\right)} & \leqslant C_{14}\left(\left\|\rho_{0}\right\|_{W_{2}^{1}(\Omega)}, M, C_{12}, C_{6}\right) \\
\left\|\frac{\partial \rho}{\partial t}\right\|_{L_{\infty}\left(0, T, L_{2}(\Omega)\right)} & \leqslant C_{15}\left(\left\|\rho_{0}\right\|_{W_{2}^{1}(\Omega)}, M, C_{12}, C_{6}\right) \\
\left\|\nabla \otimes \frac{\partial u}{\partial t}\right\|_{L_{2}\left(Q_{T}\right)} & \leqslant C_{16}\left(M, C_{13},\|f\|_{L_{2}\left(0, T, W_{2}^{1}(\Omega)\right)}\right) \\
\|\operatorname{div} \mathbb{P}\|_{L_{2}\left(0, T, W_{r}^{1}(\Omega)\right)} & \leqslant C_{17}\left(C_{6}, C_{13}, C_{14}, r\right) \quad \forall r<\frac{n}{n-1}, \quad s=\frac{4}{3+(\beta-2) \alpha / \beta} .
\end{aligned}
$$

Действительно, разделив (0.1) на $\rho$ и применив операцию $\nabla$, с учетом (1.1) получим тождество

$$
\frac{d}{d t} \nabla \ln \rho+(\nabla \otimes \boldsymbol{u}) \nabla \ln \rho+\nabla \operatorname{div} \boldsymbol{u}=0
$$


которое после умножения на $2 \nabla \ln \rho$ и интегрирования по $\Omega$ дает

$$
\begin{aligned}
& \frac{d}{d t} \int_{\Omega}|\nabla \ln \rho|^{2} d \boldsymbol{x}=\int_{\Omega}|\nabla \ln \rho|^{2} \operatorname{div} \boldsymbol{u} d \boldsymbol{x}-2 \int_{\Omega}(\mathbb{D} \nabla \ln \rho, \nabla \ln \rho) d \boldsymbol{x} \\
& \quad-2 \int_{\Omega} \nabla \operatorname{div} \boldsymbol{u} \cdot \nabla \ln \rho d \boldsymbol{x} \leqslant\left(3 M_{1}+1\right) \int_{\Omega}|\nabla \ln \rho|^{2} d \boldsymbol{x}+\int_{\Omega}|\nabla \operatorname{div} \boldsymbol{u}|^{2} d \boldsymbol{x},
\end{aligned}
$$

и теперь (4.1) очевидно ввиду $(2.20),(2.17)$ и (3.11). Оценки (4.2)-(4.4) суть элементарные следствия оценок п. $3,(4.1)$ и уравнений (0.1)-(0.3), а (4.5) вытекает из (2.13) и $(3.14)-(3.16)$.

Классы разрешимости и единственности. Пусть имеются два (гладких) решения задачи $(0.1)-(0.4),(3.5),(3.6)-\left(\rho_{1}, \boldsymbol{u}_{1}\right)$ и $\left(\rho_{2}, \boldsymbol{u}_{2}\right)$. Будем обозначать нижним индексом также все величины, связанные с соответствующими решениями. Обозначим $\rho=\rho_{1}-\rho_{2}$, $\boldsymbol{u}=\boldsymbol{u}_{1}-\boldsymbol{u}_{2}$. Для этих величин получаем следующую задачу:

$$
\begin{aligned}
& \frac{\partial \rho}{\partial t}+\operatorname{div}\left(\rho \boldsymbol{u}_{2}\right)=-\operatorname{div}\left(\rho_{1} \boldsymbol{u}\right), \\
& \frac{\partial \boldsymbol{u}}{\partial t}+\left(\boldsymbol{u}_{1} \cdot \nabla\right) \boldsymbol{u}-\frac{\operatorname{div} \mathbb{P}_{1}-\operatorname{div} \mathbb{P}_{2}}{\rho_{1}}=-(\boldsymbol{u} \cdot \nabla) \boldsymbol{u}_{2}-\frac{\rho}{\rho_{1} \rho_{2}} \operatorname{div} \mathbb{P}_{2}+\rho \boldsymbol{f}, \\
& \left.\rho\right|_{t=0}=0,\left.\quad \boldsymbol{u}\right|_{t=0}=0,
\end{aligned}
$$

плюс условие периодичности. Умножая аналог уравнения (0.1) для $\rho_{1}$ на $|\boldsymbol{u}|^{2} / 2,(4.7)$ на $\rho_{1} \boldsymbol{u}$ и складьвая, после интегрирования по $\Omega$ вьведем тождество

$$
\begin{aligned}
& \frac{d}{d t} \int_{\Omega} \frac{\rho_{1}|\boldsymbol{u}|^{2}}{2} d \boldsymbol{x}+\int_{\Omega}\left(\mathbb{P}_{1}-\mathbb{P}_{2}\right):\left(\mathbb{D}_{1}-\mathbb{D}_{2}\right) d \boldsymbol{x} \\
& \quad=\int_{\Omega}\left(-\rho \boldsymbol{u} \cdot \boldsymbol{w}_{2}-\left(\rho_{1} \boldsymbol{u},\left(\nabla \otimes \boldsymbol{u}_{2}\right)^{*} \boldsymbol{u}\right)+\rho_{1} \boldsymbol{f} \cdot \rho \boldsymbol{u}\right) d \boldsymbol{x}
\end{aligned}
$$

С другой стороны, умножая (4.6) на $2 \rho$ и интегрируя по $\Omega$, получим

$$
\frac{d}{d t} \int_{\Omega} \rho^{2} d \boldsymbol{x}=\int_{\Omega}\left(-\rho^{2} \operatorname{div} \boldsymbol{u}_{2}-2 \rho_{1} \rho \operatorname{div} \boldsymbol{u}-2 \rho \boldsymbol{u} \cdot \nabla \rho_{1}\right) d \boldsymbol{x}
$$

что с учетом $(4.8),(4.9)$ и (4.11) дает оценку

$$
\int_{\Omega}\left(\rho_{1}|\boldsymbol{u}|^{2}+\rho^{2}\right) d \boldsymbol{x} \leqslant \int_{0}^{t} \int_{\Omega} F \cdot\left(\rho_{1}|\boldsymbol{u}|^{2}+\rho^{2}\right) d \boldsymbol{x} d s
$$

где

$$
F=2\left|\mathbb{D}_{2}\right|+\frac{\rho_{1}^{2}}{2 C_{1}}+\left(1+\frac{1}{\rho_{1}}\right)\left(\left|\nabla \rho_{1}\right|+\left|\boldsymbol{w}_{2}\right|+\rho_{1}|\boldsymbol{f}|\right) .
$$

Неравенство (4.10) гарантирует $\rho=0, \boldsymbol{u}=0$, если суммируемость $F$ по $\boldsymbol{x}$ не хуже, чем $L_{M}$ (пространство Орлича) с $M(s)=e^{s}$ (см. [9]). Как видно, полученные выше оценки наиболее близки к классу единственности в случае $n=2$, когда достаточно дополнительно лишь (по аналогии с выводом (4.1) из (3.11)) “извлечь" из (3.17) оценку $\rho_{1}$ в $W_{2}^{2}(\Omega)$ (что снова сводится к третьей энергетической оценке для $(2.1)$ ). 
Разрешимость “в целом" задачи (0.1)-(0.4), (3.5), (3.6) не вызьвает сомнений по крайней мере в классах, соответствующих первым двум энергетическим оценкам (3.7)-(3.11) (и их следствию (4.1)), - доказательство может быть проведено, например, методом типа Галёркина (как в [5]). Поэтому доказательство разрешимости в классе, соответствующем всем полученньм в данной работе оценкам, представляется не столь интересной задачей (особенно ввиду того, что единственность этих решений, даже при $n=2$, остается пока “под вопросом"), и мы не будем этого делать.

Таким образом, дальнейшее продвижение в глобальной теории системы $(0.1)-(0.4)$ (существование “в целом" в классах единственности решений, вплоть до классических) связано с изучением регулярности решений системы (2.1). В связи с этим интересно отметить некоторые

Дополнительные свойства системы (2.1), (2.6). Помимо энергетических оценок мы еще располагаем их интерполящионньми следствиями. Поскольку оператор системы (2.1) нелинейньй, мы вьнуждены использовать методы нелинейной интерполяции (см. $[10$, с. 102] со ссылкой на [11]), что требует предварительно установить гёльдеровость оператора. В (2.6) каждое из слагаемых порождает монотонньй оператор

$$
\operatorname{div} \frac{\partial V(\cdot)}{\partial \mathbb{D}}
$$

(т.е. обладающий свойством $(2.5)_{2}$ ), а слагаемое с $\Gamma_{1}$ еще и строго монотонньй (см. [4]) при условии (2.18), так что

$$
\left(\frac{\partial V}{\partial \mathbb{D}}\left(\mathbb{D}_{2}\right)-\frac{\partial V}{\partial \mathbb{D}}\left(\mathbb{D}_{1}\right)\right):\left(\mathbb{D}_{2}-\mathbb{D}_{1}\right) \geqslant C_{1}\left|\mathbb{D}_{2}-\mathbb{D}_{1}\right|^{2}
$$

где $\mathbb{D}_{i}=\mathbb{D}\left(\boldsymbol{u}_{i}\right)$. Отметим, что если $(2.18)$ не вьполнено, но $\Gamma_{2}(\xi)=\beta \xi, \beta=$ const $>0$, то

$$
\left(\frac{\partial V}{\partial \mathbb{D}}\left(\mathbb{D}_{2}\right)-\frac{\partial V}{\partial \mathbb{D}}\left(\mathbb{D}_{1}\right)\right):\left(\mathbb{D}_{2}-\mathbb{D}_{1}\right) \geqslant \frac{\beta}{4 n}\left|\mathbb{D}_{2}-\mathbb{D}_{1}\right|^{4}
$$

В самом деле, (4.12) следует из соотношений

$$
\left(\mathbb{A}^{3}-\mathbb{B}^{3}\right):(\mathbb{A}-\mathbb{B})=\frac{1}{4}\left|(\mathbb{A}-\mathbb{B})^{2}\right|^{2}+\frac{3}{4}\left|\mathbb{A}^{2}-\mathbb{B}^{2}\right|^{2} \geqslant \frac{1}{4 n}|\mathbb{A}-\mathbb{B}|^{4},
$$

справедливых для всех симметричных тензоров $\mathbb{A}$ и $\mathbb{B}$.

Продемонстрируем интерполяционные свойства системы $(2.1),(2.6)$ для определенности в следующей частной ситуации:

$$
n=3, \quad \Lambda(\xi)=\mathrm{const} \cdot \xi, \quad \Gamma_{s}(\xi)=\mathrm{const} \cdot \xi, \quad s>1, \quad \Gamma_{1}(\xi)=(1+\xi)^{p}, \quad p \geqslant 1 .
$$

Будем рассматривать оператор $T$, осуществляющий в (2.1) соответствие

$$
T: \mathbf{F} \mapsto \mathbb{D}(\boldsymbol{u})
$$

Первая априорная оценка (следствие (2.14)) принимает вид

$$
\|T(\mathbf{F})\|_{L_{2 p}(\Omega)} \leqslant C_{19}(p, \Omega)\|\mathbf{F}\|_{W_{2 p /(2 p-1)}^{-1 /(2 p-1)}(\Omega)}^{1 /},
$$


а вторая оценка (2.20) дает

$$
\left\|\nabla\left(1+|\mathbb{D}|^{2}\right)^{p / 2}\right\|_{L_{2}(\Omega)} \leqslant C_{20}(p, \Omega)\|\mathbf{F}\|_{L_{2}(\Omega)},
$$

что вместе с (4.14) позволяет оценить $|\mathbb{D}|^{p}$ в $W_{2}^{1}(\Omega)$ и после применения вложения $W_{2}^{1}$ в $L_{6}$ записать

$$
\|T(\mathbf{F})\|_{L_{6 p}(\Omega)} \leqslant C_{21}(p, \Omega)\left(\|\mathbf{F}\|_{W_{2 p /(2 p-1)}^{-1 /(2 p-1)}}^{1 /(2)}+\|\mathbf{F}\|_{L_{2}(\Omega)}^{1 / p}\right) .
$$

Неравенства (4.11) и (4.12) очевидньм образом дают оценки гёльдеровости

$$
\begin{aligned}
& \left\|T\left(\mathbf{F}_{1}\right)-T\left(\mathbf{F}_{2}\right)\right\|_{L_{2}(\Omega)} \leqslant \frac{1}{C_{1}}\left\|\mathbf{F}_{1}-\mathbf{F}_{2}\right\|_{W_{2}^{-1}(\Omega)}, \\
& \left\|T\left(\mathbf{F}_{1}\right)-T\left(\mathbf{F}_{2}\right)\right\|_{L_{4}(\Omega)} \leqslant\left(\frac{4 n}{\beta}\right)^{1 / 3}\left\|\mathbf{F}_{1}-\mathbf{F}_{2}\right\|_{W_{4 / 3}^{-1}(\Omega)}^{1 / 3}
\end{aligned}
$$

соответственно (рассмотрим для определенности случай (4.16)). Следуя обозначениям и утверждениям из [10, с. 102-103], применим к оператору $T$ интерполяционную теорему Л. Тартара (т.е. проинтерполируем между (4.14) и (4.15), что возможно благодаpя (4.16)) c

$$
A_{0}=L_{6 / 5}(\Omega), \quad B_{0}=L_{2}(\Omega), \quad A_{1}=L_{2}(\Omega), \quad B_{1}=L_{6 p}(\Omega), \quad \alpha_{0}=1, \quad \alpha_{1}=\frac{1}{p} .
$$

Получим

$$
T:\left(A_{0}, A_{1}\right)_{\eta, r} \rightarrow\left(B_{0}, B_{1}\right)_{\theta, q}
$$

где

$$
\theta \in(0,1), \quad q \geqslant 1, \quad \eta=\frac{\theta}{p-(p-1) \theta}, \quad r=\frac{p-(p-1) \theta}{p} q .
$$

Напомним $[10$, с. 17,142$]$, что $\left(L_{a}, L_{b}\right)_{c, d}=L_{(a, b)_{c}, d}$ при $a<b, a<d$, где $\left((a, b)_{c}\right)^{-1}=$ $c b^{-1}+(1-c) a^{-1}$, а $L_{\alpha, \beta}$ обозначает пространства Лоренца, от которых мы избавимся, воспользовавшись следующими их свойствами:

$$
L_{\alpha, \beta} \hookrightarrow L_{\alpha, \gamma} \text { при } \beta \leqslant \gamma, \quad L_{\alpha, \alpha}=L_{\alpha} .
$$

Итак, несколько “загрубив” (4.17), перепишем это соотношение в терминах пространств Лебега:

$$
T: L_{(6 / 5,2)_{\eta}}(\Omega) \rightarrow L_{(2,6 p)_{\theta}}(\Omega),
$$

что возможно при условиях

$$
r \geqslant\left(\frac{6}{5}, 2\right)_{\eta}, \quad(2,6 p)_{\theta} \geqslant q .
$$

Таким образом, взяв произвольно $\theta \in(0,1)$ и вычислив $\eta$ из $(4.18)$, мы должны подобрать $q$ (а вместе с ним и соответствующее $\eta$ согласно (4.18)) так, чтобы было выполнено (4.20). Нетрудно проверить, что такое $q$ всегда найдется. В итоге мы можем сформулировать искомое соотношение (подсчитав показатели в (4.19))

$$
T: L_{A}(\Omega) \rightarrow L_{B}(\Omega), \quad \frac{6}{5} \leqslant A \leqslant 2, \quad B=\frac{3}{2} \cdot \frac{(5 p-3) A-6(p-1)}{3-A} .
$$

В заключение отметим, что если сохранить во второй априорной оценке первые производные $T(\mathbf{F})$ (а не применять вложение в $L_{6}$, как мы сделали при вьводе (4.15)), то в (4.21) получится оценка дробных производных от $\mathbb{D}$. 


\section{СПИСОК ЦИТИРОВАННОЙ ЛИТЕРАТУРЫ}

[1] Серрин Дж. Математические основы классической механики жидкости. М.: ИЛ, 1963.

[2] Вайгант В. А., Кажихов А. В. О существовании глобальных решений двумерных уравнений Навье-Стокса сжимаемой вязкой жидкости // Сиб. матем. ж. 1995. Т. 36. №6. C. $1283-1316$.

[3] Lions P.-L. Mathematical Topics in Fluid Mechanics. V. 2. Compressible Models. Oxford: Clarendon Press, 1998.

[4] Malek J., Necas J., Rokyta M., Ruzicka M. Weak and Measure-Valued Solutions to Evolutionary PDEs. London, Weinheim etc.: Chapman \& Hall, 1996.

[5] Мамонтов А. Е. Существование глобальных решений многомерных уравнений Бюргерса сжимаемой вязкой жидкости // Докл. РАН. 1998. Т. 361. № 2. С. 161-163.

[6] Мамонтов А. Е. О глобальной разрешимости многомерных уравнений Навье-Стокса сжимаемой нелинейно вязкой жидкости. I // Сиб. матем. ж. 1999. Т. 40. № 2. С. 408-420.

[7] Мамонтов А. Е. О глобальной разрешимости многомерных уравнений Навье-Стокса сжимаемой нелинейно вязкой жидкости. II // Сиб. матем. ж. 1999. Т. 40. №3. С. 635-649.

[8] Kaplický P., Malek J., Stará J. $C^{1, \alpha}$-Solutions to a Class of Nonlinear Fluids in Two Dimensions-Stationary Dirichlet Problem // Sonderforschungsbereich 256 nichtlineare partielle Differentialgleichungen. № 529. Bonn: Rheinische Friedrich-Wilhelms-Universität, 1997.

[9] Кажихов А. В., Мамонтов А. Е. Об одном классе выпуклых функций и точных классах корректности задачи Коши для уравнения переноса в пространствах Орлича // Сиб. матем. ж. 1998. Т. 39. № 4. С. 831-850.

[10] Берг Й., Лефстрем Й. Интерполяционные пространства. Введение. М.: Мир, 1980.

[11] Tartar L. Interpolation nonlineaire et regularité // J. Funct. Anal. 1972. № 9. P. 469-489.

Институт гидродинамики им. М. А. Лаврентьева СО РАН

Поступило

E-mail: mamontoff@hydro.nsc.ru

20.07.1999 